\title{
Comparative genomics of heat shock proteins system in extremophile nonbiting midges
}

\author{
Olga Kozlova \\ Kazan Federal University, Kazan, \\ Russia \\ olga-sphinx@yandex.ru
}

\author{
Guzel Gazizova \\ Kazan Federal University, Kazan, \\ Russia \\ grgazizova@gmail.com \\ Elena Shagimardanova \\ Kazan Federal University, Kazan, \\ Russia \\ rjuka@mail.ru
}

\author{
Oleg Gusev \\ Kazan Federal University, Kazan, \\ Russia \\ gaijin.ru@gmail.com
}

\begin{abstract}
Here we compare the number and expression profiles of HSP-coding genes in larvae of Chironomidae family (Diptera), who are known for their ability to successfully combat abiotic stresses using wide range of behavioral, morphological and biochemical features. In order to perform comparative studies we sequenced and assembled genomes of 4 chironomids from different habitats and also sequenced whole-genome RNA of their larvae in control and stressed conditions. It was shown that compact genome sizes (up to $200 \mathrm{Mb}$ ) are typical for Chironomidae, while changes in size of a genome are mediated by elongation and shortening of introns length, as well as by changes in quantity and content of dispersed repeats. For all extremophile species under consideration species-specific gene expansion accompanied by formation of compact clusters in a genome was detected. The most amplitudinous reaction towards abiotic stress (desiccation) was shown by anhydrobiotic species Polypedilum vanderplanki (Africa). As for HSP-coding genes, it was noticed that genes of HSP20 and HSP70 show the most dramatic and universal up-regulation of expression in response to abiotic stress, while genes of chaperonins (HSP60) tend to be up-regulated in response to desiccation, but not to heat shock. But the most surprising notion was linked to acid-tolerant species Polipedilum cf. tamanigrum (Japan), because none of HSP-coding genes in this species showed statistically significant up-regulation, what may be explained by absence of special regulatory sequence - heat shock element (HSE) in their promotor regions.
\end{abstract}

Keywords - Chironomidae, stress exposure, heat shock proteins, desiccation, genome assembly, transcriptomics

Motivation and aim

\section{Motivation}

Most abiotic stresses - such as elevated temperature, desiccation or chemical stress - lead to denaturation and aggregation of wrongly folded proteins. In order to protect their proteins from aggregation and non-native folding, all living systems use wide range of molecular chaperones, including heat shock proteins (HSPs) which present the largest and the most evolutionary conservative class of such chaperones [1]. The regulation of HSPs-coding genes in eukaryotes is performed by specific family of transcription factors - heat shock factors (HSFs), which bind to special regulatory sequence - heat shock element (HSE) in the promotor region of a target gene. Until recently the most well studied aspects of HSP system and its role in adaptation among Diptera belonged to the model genus Drosophila, whose species differ by the level of thermal adaptation and thus can be a convenient object for studying adaptations to elevated temperatures. However, it's well known that HSPs take part in the reaction towards wide range of abiotic stresses - not only to heat shock itself. Taken this into account, it could be interesting to compare features of quantitative composition, genome location and expression patterns of HSPs-coding genes in species, which are remote enough from each other to the evolutionary and geographic point of view, but united by habitation in ecological niches, linked to different extreme impacts. To this point of view the Chironomidae (non-biting midges) is considered to be one of the most perspective family of Diptera, because many of its species during their larval stage of development face need to vitality preservation in different extreme and unstable conditions, for what they use specific behavioral, physiologic and molecular-genetic adaptations [2].

Aim

The development of DNA and RNA sequencing technologies opens up broad prospects for studying features of extremophiles lives on the whole-genome level. Using bioinformatic methods of comparative genomics and transcriptomics one can find new genes, predict their functions and study changes of expression during adaptations to extreme conditions. Taking this into account, the aim of the given study was to compare the structure of de-novo assembled genomes of extremophile nonbiting midges and to characterize HSP-coding genes and their expression under abiotic stresses.

\section{Methods}

In the given research three draft genome assemblies of extremophile Chironomidae species were performed. In order to investigate changes in expression levels of HSPs-coding genes we also sequenced RNA of larvae in different stress conditions - heat shock and desiccation for Australian larvae of Paraborniella tonnoiri, heat shock for Russian larvae of «Orthocladiinae acuticauda» and heat shock and ion stress (exposition in fresh water) for acid-tolerant larvae of Polypedilum cf. tamanigrum from Japan. We also performed brand new variant of genome assembly for unique anhydrobiotic African species Polypedilum vanderplanki by sequencing DNA of Pv11 cell line, derived from embryonic mass of the insect [3]. For differential expression analysis of genes of $P$. vanderplanki we used earlier sequenced libraries, which reflect desiccation-rehydration cycle of its larvae [4]. For orthogroups searching we used all predicted aminoacid sequences of species under consideration as well as proteins for 6 other Diptera. 


\section{Results}

It was found that in genomes of species, which occupy similar ecological niches, even if they are remote from each other geographically and evolutionary, specific orthogroups, which are either totally unique for the species or have significant increase in copy number, can be found. This observation becomes clearer in case of species, which face desiccation in natural habitats, even despite the differences in their physiological, morphological and behavioral adaptations. Thus, a presence of convergent evolutionary changes in genome structure as a result of colonization of new ecological niches, extreme environments of which led to appearance of similar molecular-genetic adaptations could be suggested. Another typical trait of genome structure of extremophile chironomids is compact clusters of paralogous genes, whose expression simultaneously increases after exposure to stress. Among the most obvious examples of this observation there are genes, coding for cysteine-rich secretory proteins of CRISP family in "Orthocladiinae acuticauda» genome (12 gene copies against maximum 4 in other genomes including model Diptera). The compact cluster structures in a genome are also typical for some genes, coding for small HSPs. In contrast to high-molecular HSPs, the number of these genes differs between species: from 10 copies for $P$. $c f$. tamanigrum till 15 copies for $P$. vanderplanki. It was shown that each extremophile species (except for $P$. cf. tamanigrum) has its own specific cluster with expansion of gene copies, and exactly these species-specific genes show sharp increase of expression after stress. This allows suggesting that the adaptation to extreme environments by using small HSPs walked the path of gene expansion as well as regulation. Among all HSPs-coding genes those corresponding to lowmolecular HSP and HSP70 families showed the most dramatic and universal up-regulation of expression in response to abiotic stresses. In contrast to other extremophile species, in case of desiccation-tolerant midge $P$. vanderplanki we noted up-regulation of almost each HSP-coding gene (except for some low-molecular HSPs). Chaperonins (HSP60 family) were specifically up-regulated in case of desiccation, but not after heat shock. But the most surprising observation was linked to acid-tolerant species $P$. cf. tamanigrum which showed significant up-regulation of HSPs-coding genes neither after heat shock nor after ion stress. In order to find a possible reason of this notion we performed motif enrichment analysis in supposed promotor regions of HSPs-coding genes, taking 500 random genes of each genome as a control group. As a result we found statistically significant enrichment of motifs, similar to Drosophila's HSE for genomes of «Orthocladiinae acuticauda», Paraborniella tonnoiri and Polypedilum vanderplanki, but no enrichment for Polypedilum cf. tamanigrum. Thus we could make a conclusion that all HSP system is not involved in reaction to heat shock in P. cf. tamanigrum, though for the present it is not clear, how such conservative mechanism of regulation could have been broken.

\section{Acknowledgment}

The work was performed according to the Russian Government Program of Competitive Growth of Kazan Federal University.

\section{References}

[1] Chen B. et al. (2018) Evolution of heat-shock protein expression underlying adaptive responses to environmental stress. Molecular Ecology. 27(15): 3040-3054.

[2] Cornette R. et al. (2015) Chironomid midges (Diptera, chironomidae) show extremely small genome sizes. Zoological science. 32(3): 248-254.

[3] Nakahara Y. et al. (2010) Cells from an anhydrobiotic chironomid survive almost complete desiccation. Cryobiology. 60(2): 138146.

[4] Gusev O. et al. (2014) Comparative genome sequencing reveals genomic signature of extreme desiccation tolerance in the anhydrobiotic midge. Nature Communications. 5: 4784 